\title{
Continuous Estimation of Stress Using Physiological Signals during a Car Race
}

\author{
Wen Wen', Daisuke Tomoi ${ }^{1}$, Hiroshi Yamakawa ${ }^{1}$, Shunsuke Hamasaki ${ }^{1}$, Kaoru Takakusaki ${ }^{2}$, \\ Qi An ${ }^{1}$, Yusuke Tamura ${ }^{1}$, Atsushi Yamashita1, Hajime Asama1 \\ ${ }^{1}$ Department of Precision Engineering, University of Tokyo, Tokyo, Japan \\ ${ }^{2}$ The Center for Brain Function and Medical Engineering, Asahikawa Medical College, Asahikawa, Japan \\ Email:wen@robot.t.u-tokyo.ac.jp
}

How to cite this paper: Wen, W., Tomoi, D., Yamakawa, H., Hamasaki, S., Takakusaki, K., An, Q., Tamura, Y., Yamashita, A., \& Asama, H. (2017). Continuous Estimation of Stress Using Physiological Signals during a Car Race. Psychology, 8, 978-986. https://doi.org/10.4236/psych.2017.87064

Received: April 5, 2017

Accepted: May 16, 2017

Published: May 19, 2017

Copyright (c) 2017 by authors and Scientific Research Publishing Inc. This work is licensed under the Creative Commons Attribution International License (CC BY 4.0).

http://creativecommons.org/licenses/by/4.0/ c) (i) Open Access

\begin{abstract}
Mental stress refers to the feeling of strain and anxiety caused by internal and/or external factors. Stress may have both positive and negative influences on cognitive performance. For example, small amounts of stress may improve concentration, athletic performance, and reaction speed. However, excessive stress may harm health, and disturb concentration and motor control. Furthermore, increase in short-term stress has a considerable effect on physiological processes such as heart rate and sweating. However, to our knowledge, so far no study has estimated mental stress through continuous monitoring of physiological signals under a situation with various stressors. Therefore, it remains unclear how different physiological signals correlate with each other and how they change continuously in response to the changes in stressors. Here, we measured heart rate variability, galvanic skin response, and activity of the masseter muscle in a professional racer during a real car race. Car racing is one of the most dangerous sports, and the competition with other racers and physical discomfort during the race induce great short-term stresses. We used factor analysis to examine the relation between the three types of physiological signals, and clarified the events associated with stress (e.g., acceleration, competing car in vision, overtaking). The results showed that the heart rate variability and galvanic skin response correlated with each other, and were associated with the event of competition, which brought great mental stress. In contrast, activity of the masseter muscle did not correlate with the other two physiological signals, but was associated with the events of acceleration or deceleration, which brought great physical discomfort. We concluded that heart rate variability and galvanic skin response reflect mental stress, which is associated with an internal desire to win and is triggered by changes in the external world (e.g., the appearance of competing cars). In contrast, masseter muscle activity reflects the endurance of body discomfort, which is not linked to any internal state. Our results indicated that internal and external stressors may be dissociated from each other, and may affect different or-
\end{abstract}


gans and physiological receptors.

\section{Keywords}

Stress, Physiological Signals, Heart Rate, Galvanic Skin Response, Masseter Muscle Activity, Factor Analysis

\section{Introduction}

In psychology, stress refers to the mental state of feeling strain and anxiety, and is usually caused by changes in the environment or internal desire. Small amounts of stress could be helpful to improve concentration and athletic performance. However, excessive or chronic stress usually causes depression, harms health, and results in errors in decision-making or motor control. There are many types of stressors, such as crises, threats, daily annoyances, and life events. In the present study, we focused on short-term stress, which arises from rapid environmental changes such as threat, cognitive load, and emotions.

Stress is a significant subjective experience that is difficult to estimate quantitatively from a subjective perspective. An individual may be able to report whether he/she is undergoing great or small stress, but is not able to quantify it, or to compare the amount of stress between different stressors. However, previous studies have attempted to measure physiological reactions such as heartbeat, sweating, muscle activities, and blood pressure in stressful situations as an approach to detect or estimate the amount of stress (Hidaka, Yanagi, \& Takada, 2004b; Hjortskov et al., 2004; Kurniawan, Maslov, \& Pechenizkiy, 2013; Shi et al., 2007; Thayer, Ahs, Fredrikson, Sollers, \& Wager, 2012; Yemm, 1969). Previous studies have revealed useful links between some physiological signals and stress. However, none of these studies has examined physiological signals in a situation with multiple and dynamically changing stressors.

In the present study, we focused on the situation of a real car race. We continuously measured three types of physiological signals in a professional driver during a real race. Car racing is one of the most dangerous sports, and requires continuous and rapid decisions during a relatively long duration. Both the competition and physical discomfort could be stressors, and multiple stressors may change rapidly. The present study aimed to clarify the relations between multiple physiological signals (i.e. heartbeat, sweating, and masseter muscle activity) in such a situation, and to elucidate the relation between physiological signals and different stressors. Our present findings could not only provide useful estimation of stress during the specific situation of car race but also contribute to the understanding of the subjective experience of stress.

\section{Methods}

\subsection{Participant and Experimental Environment}

The present study focused on within-individual correlation in a highly specific 
situation. Therefore, we invited a professional race driver (male, age 27 years) to take part in a real car race, and measured physiological signals during the race. The study was approved by the ethics committee of the Faculty of Engineering at the University of Tokyo, and written informed consent was obtained from the participant prior to the experiment.

The experiment was performed during a practice session of a race, in which all racers took part in order to get used to the racing course in advance of the actual race. The racing condition was the same as the actual one, in which the racers were free to overtake other cars. Figure 1 illustrates the map of the racing course of the present study. The racing course contained 12 corners and two slopes, and therefore required highly precise control of speed and direction.

\subsection{Equipment}

The present study measured the participant's heart rate variability (HRV), galvanic skin response (GSR), and muscle activity of the masseter (MAM). All three physiobiological signals have been reported to be associated with stress (Hidaka et al., 2004b; Hjortskov et al., 2004; Kurniawan et al., 2013; Shi et al., 2007; Thayer et al., 2012; Yemm, 1969). However, previous studies used different signals in different stressful conditions, and the relation between these signals and different stressors remains unknown.

The three physiobiological signals were measured using galvanic skin response (GSR) sensors (DL-340, S\&ME), heartbeat (electrocardiogram [ECG]) sensors (DL-310, S\&ME), and electromyography (EMG) sensors (DL-41, S\&ME), and were recorded in a data logger (DL-3100, S\&ME), which was attached to the participant's waist. The sensors were attached to the participant's skin using medical tape, and were referenced to the right wrist. Figure 2 illustrates the measuring positions of all three signals.

\subsection{Preprocessing of the Physiobiological Signals}

All three physiobiological signals were recorded at a frequency of $1000 \mathrm{~Hz}$. The heart rate variability was recorded from ECG electrodes, which were placed at

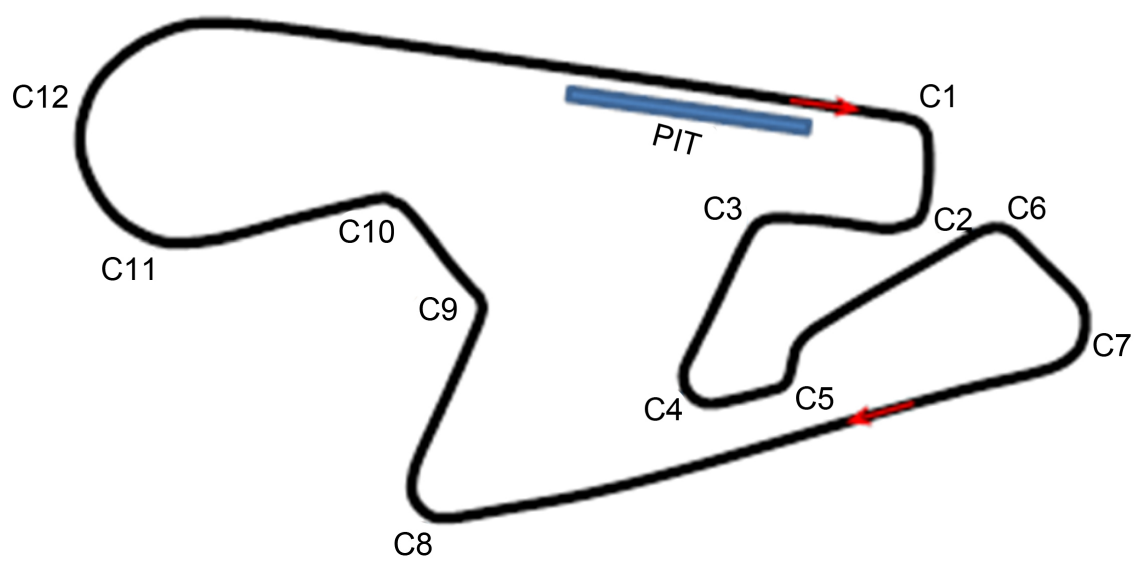

Figure 1. Racing course used in the experiment. 


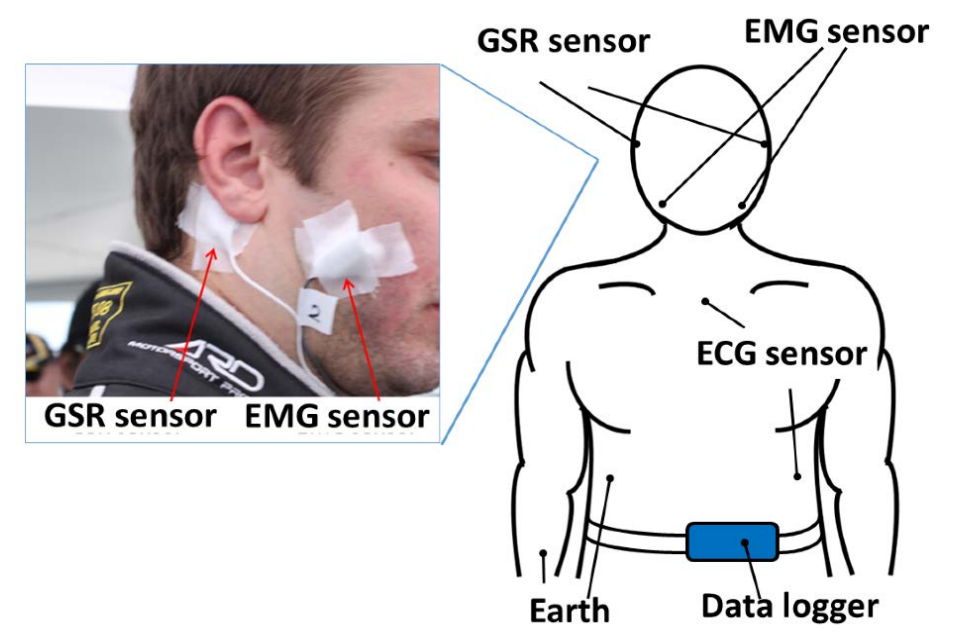

Figure 2. Positions of the sensors. GSR, galvanic skin response; EMG, electromyogram (to measure muscle activities); ECG, electrocardiogram (to measure heart rate).

the distal part of the sternum and at the sixth rib in the left axilla. An offline band filter of $0.16-500 \mathrm{~Hz}$ was applied to the ECG signals. From the ECG, we calculated the intervals between peaks (R-R intervals), standard deviation of the $\mathrm{R}-\mathrm{R}$ intervals (SDNN), and root mean squared successive differences of R-R intervals (RMSSD). The ratio of SDNN to RMSSD was used as the index of HRV. The GSR was recorded using GSR sensors placed at the left and right mastoids. Most previous studies placed the GSR sensors on the hand or foot (e.g., Healey \& Picard, 2000; Nourbakhsh, Wang, Chen, \& Calvo, 2012). However, in the real car racing, this may disturb the driver's sensation on hands and feet, and has the risk of causing fatal mistakes. Therefore, we avoided these positions and chose mastoids as the measuring site instead. Finally, the MAM was recorded from two pairs of EMG sensors placed at the left and right masseters. Activities of facial muscles are useful objective indices of emotion (Cacioppo, Petty, Losch, \& Kim, 1986), and jaw muscles in particular are susceptible to mental stress (Hidaka, Yanagi, \& Takada, 2004a; Hidaka et al., 2004b). However, unlike HRV and GSR, masseters can be activated by consciousness or habit; therefore, the role of the MAM sensor as an anchor of stress may be different from the former two sensors.

\subsection{Procedure}

Physiological signals were recorded during the practice session of a real race. The participant raced over the entire racing course six laps without break. The race was recorded with a video camera that was installed in the racing car and recorded the view of the driver. The position of the participant's racing car was recorded using global positioning system (GPS) sensors attached to the car.

\section{Results}

According to the results of an interview with the participant, the first and second 
laps were considered as a warming up phase and were therefore excluded from the analysis. Only the results from the third and sixth laps were included in the analysis. We used factor analysis to examine the relations between the three physiological signals. The analysis revealed two factors: the first factor 1) influenced both HRV and GSR, while the second factor 2) influenced MAM. Table 1 summarizes the coefficients of the two factors. The factor coefficient shows the extent to which a factor influences a variable.

In order to determine the events that were associated with the observed activities of the two factors, we abstracted two events from the video and GPS records of the car: 1) competition and 2) acceleration/deceleration. The event of competition refers to the time when another car appeared in the visual field of the participant (Figure 3). The event of acceleration/deceleration was calculated from the GPS recordings. Figure 4 illustrates the plots of the factor scores in function of time. The events of competition greatly overlaid with the high scores of factor 1 (i.e. HRV and GSR), while the events of acceleration/deceleration greatly overlaid with high scores of factor 2 (i.e. MAM). Furthermore, a qualitative analysis showed that $92 \%$ of the events of competition consisted of high scores of factor 1 ( $>2.5$ standard deviation), but only $37 \%$ contained high scores of factor 2 . In contrast, $54 \%$ of the events of acceleration/deceleration contained high scores of factor 1 , while $63 \%$ contained high scores of factor 2 . In summary, the results showed that factor 1 influenced HRV and GSR and was associated with the event of competition, while factor 2 influenced MAM and was associated with the event of acceleration/deceleration.

Table 1. Coefficients of the two factors.

\begin{tabular}{ccc}
\hline & Factor 1 & Factor 2 \\
\hline HRV & 0.362 & 0.154 \\
GSR & 0.346 & -0.207 \\
MAM & 0.015 & 0.355 \\
\hline
\end{tabular}

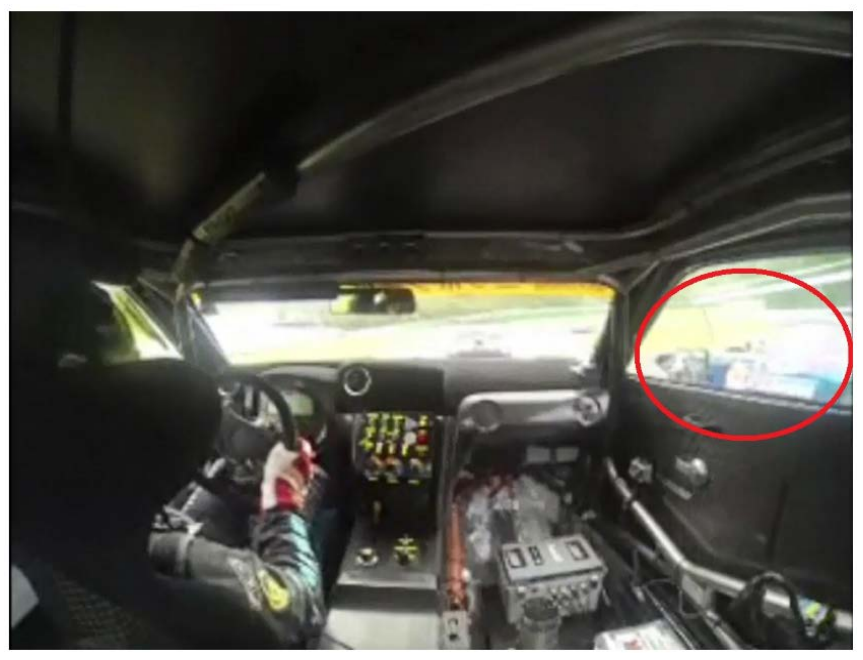

Figure 3. An example of a competition event. 

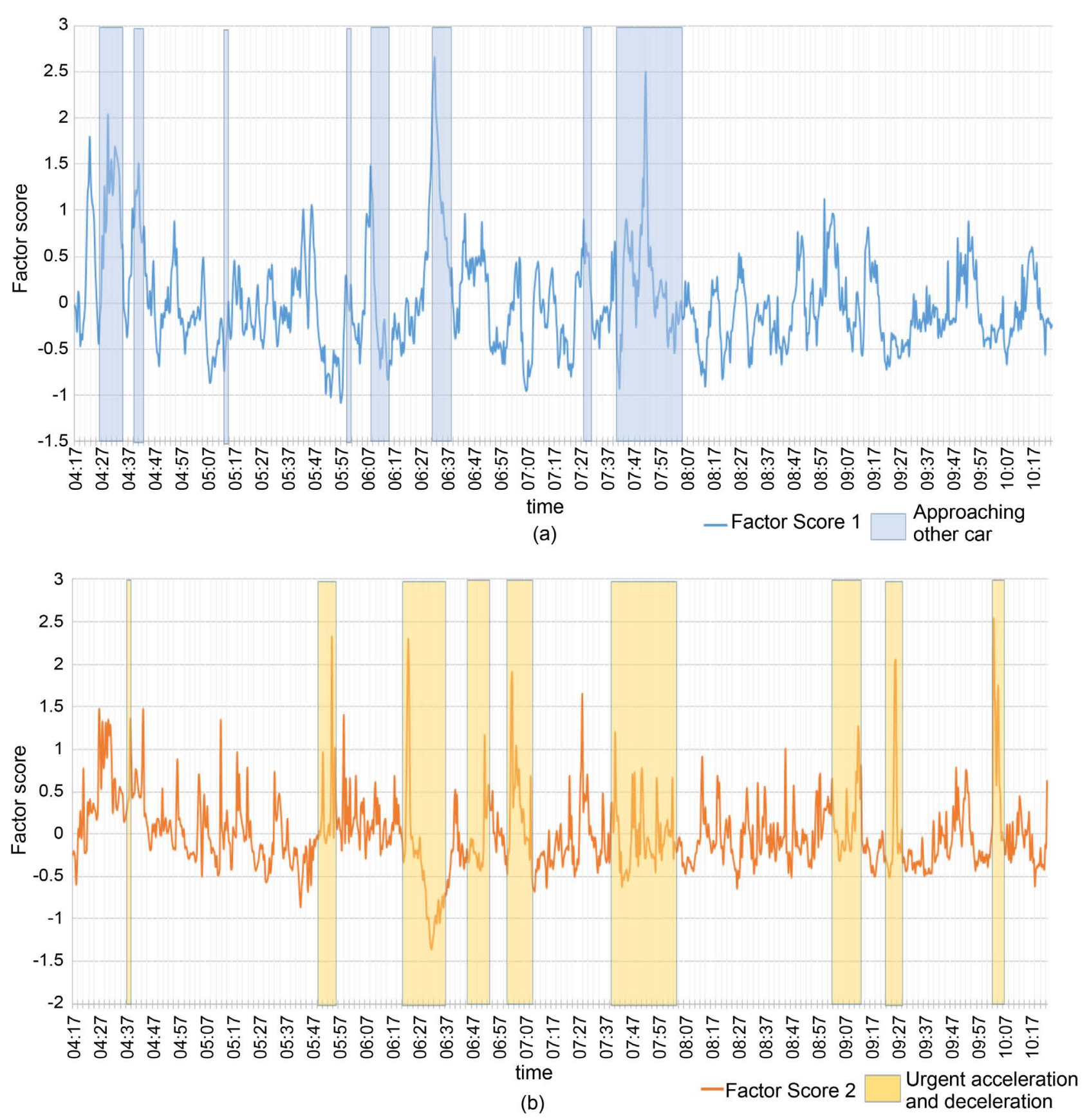

Figure 4. The scores of factor 1 (a) and 2 (b) plotted in function of time, and the overlay with the events of competition and acceleration/deceleration.

\section{Discussion}

The present study measured three different physiological signals, all of which are considered to reflect mental stress, in a professional driver during a real car race. We examined the relations between the three physiological signals using factor analysis, and the association with significant events during the race. The results showed that HRV and GSR were highly correlated with each other, and were associated with the events of competition. On the other hand, MAM was not correlated with the other two physiological signals, and was associated with the 
events of acceleration/deceleration. Our present findings suggested that, although all the three physiological signals are somehow affected by stress, they probably reflect different types of stressors. In particular, HRV and GSR are probably closer to mental stress, which is mainly the result of internal state of desire and triggered by changes in the external world. On the other hand, MAM may be triggered by physical discomfort, which could also result in stress, but is more bottom-up and does not require an internal state.

To our knowledge, the present study is the first that measured multiple physiological signals from an extremely dangerous and stressful situation in real life. Most previous studies examined stress under experimental conditions, and the "real" stress may have not been accurately simulated. The stressors examined in previous studies were more controlled and had less variability. For example, cognitive load (Hjortskov et al., 2004; Lattimore \& Maxwell, 2004; Shi et al., 2007), unfriendly attitude (Hjortskov et al., 2004; Montirosso, Provenzi, Calciolari, Borgatti, \& Group, 2011), and emotion (Kim, Bang, \& Kim, 2004) are the most common stressors used in experimental tasks. However, none of them provides continuous and diverse changes in stress, and therefore could not reveal the relationship between different physiological signals during such changes. During the car race, the driver was not only under high cognitive load but also faced a life-threatening situation, and was inspired by the desire to win at every moment. In addition, because of the high velocity and g-force during acceleration/deceleration, the driver also suffered from stress resulting from physical discomfort. Therefore, the situation of car racing provided an ideal environment of multiple sources of stress and continuous changes, allowing us to examine the continuous changes in physiological reactions that are associated with different stressors.

The most important finding of our results was that stressors associated with an internal state, such as the desire to win, and that are only linked to external stimuli may result in different physiological reactions. During the race, acceleration/deceleration brought discomfort to the body because of large g-force, and probably resulted in a stressful situation. However, both HRV and GSR were not sensitive to such condition, indicating that, although the participant might be suffering from a physical discomfort, his mental stress associated with the interval state did not change significantly. This does not necessarily mean that the mental stresses resulted from internal and external states have to be dissociated. Instead, in most occasions, they might be linked to each other, but learning (e.g., long-term exposure to a specific physical discomfort) may be able to separate them, thus controlling mental stress from the bottom-up physical stimuli.

\section{Conclusion}

The present study showed that competition and physical discomfort may result in different physiological reactions during a real car race. In particular, we found that the event of competition triggered an increase in HRV and GSR, while the acceleration/deceleration event triggered MAM. The results indicated that dif- 
ferent stressors, associated with internal or external states or stimuli, may result in disparate physiological reactions.

\section{Acknowledgements}

This work was supported in part by JSPS KAKENHI (Grant Numbers 26120005).

\section{References}

Cacioppo, J. T., Petty, R. E., Losch, M. E., \& Kim, H. S. (1986). Electromyographic Activity over Facial Muscle Regions Can Differentiate the Valence and Intensity of Affective Reactions. Journal of Personality and Social Psychology, 50, 260-268.

https://doi.org/10.1037/0022-3514.50.2.260

Healey, J., \& Picard, R. (2000). SmartCar: Detecting Driver Stress. Proceedings 15th International Conference on Pattern Recognition. ICPR-2000, 4, 218-221. https://doi.org/10.1109/ICPR.2000.902898

Hidaka, O., Yanagi, M., \& Takada, K. (2004a). Changes in Masseteric Hemodynamics Time-Related to Mental Stress. Journal of Dental Research, 83, 185-190. https://doi.org/10.1177/154405910408300220

Hidaka, O., Yanagi, M., \& Takada, K. (2004b). Mental Stress-Induced Physiological Changes in the Human Masseter Muscle. Journal of Dental Research, 83, 227-231. https://doi.org/10.1177/154405910408300308

Hjortskov, N., Rissén, D., Blangsted, A. K., Fallentin, N., Lundberg, U., \& Søgaard, K. (2004). The Effect of Mental Stress on Heart Rate Variability and Blood Pressure during Computer Work. European Journal of Applied Physiology, 92, 84-89.

https://doi.org/10.1007/s00421-004-1055-Z

Kim, K. H., Bang, S. W., \& Kim, S. R. (2004). Emotion Recognition System Using Short-Term Monitoring of Physiological Signals. Medical and Biological Engineering and Computing, 42, 419-427. https://doi.org/10.1007/BF02344719

Kurniawan, H., Maslov, A. V., \& Pechenizkiy, M. (2013). Stress Detection from Speech and Galvanic Skin Response Signals. Proceedings of CBMS 2013-26th IEEE International Symposium on Computer-Based Medical Systems, 209-214.

https://doi.org/10.1109/cbms.2013.6627790

Lattimore, P., \& Maxwell, L. (2004). Cognitive Load, Stress, and Disinhibited Eating. Eating Behaviors, 5, 315-324. https://doi.org/10.1016/j.eatbeh.2004.04.009

Montirosso, R., Provenzi, L., Calciolari, G., Borgatti, R., \& Group, N.-A. S. (2011). Measuring Maternal Stress and Perceived Support in 25 Italian NICUs. Acta Paediatrica, 101, 136-142. https://doi.org/10.1111/j.1651-2227.2011.02440.x

Nourbakhsh, N., Wang, Y., Chen, F., \& Calvo, R. A. (2012). Using Galvanic Skin Response for Cognitive Load Measurement in Arithmetic and Reading Tasks. Proceedings of the 24th Conference on Australian Computer-Human Interaction OzCHI'12, 420-423. https://doi.org/10.1145/2414536.2414602

Shi, Y., Park, T., Ruiz, N., Taib, R., Choi, E. H. C., \& Chen, F. (2007). Galvanic Skin Response (GSR) as an Index of Cognitive Load. CHI EA'O7 CHI'O7 Extended Abstracts on Human Factors in Computing Systems, 2651-2656. https://doi.org/10.1145/1240866.1241057

Thayer, J. F., Ahs, F., Fredrikson, M., Sollers, J. J., \& Wager, T. D. (2012). A Meta-Analysis of Heart Rate Variability and Neuroimaging Studies: Implications for Heart Rate Variability as a Marker of Stress and Health. Neuroscience and Biobehavioral Reviews, 36, 747-756. https://doi.org/10.1016/j.neubiorev.2011.11.009 
Yemm, R. (1969). Variations in the Electrical Activity of the Human Masseter Muscle Occurring in Association with Emotional Stress. Archives of Oral Biology, 14, 873-878. https://doi.org/10.1016/0003-9969(69)90265-9

Submit or recommend next manuscript to SCIRP and we will provide best service for you:

Accepting pre-submission inquiries through Email, Facebook, LinkedIn, Twitter, etc. A wide selection of journals (inclusive of 9 subjects, more than 200 journals)

Providing 24-hour high-quality service

User-friendly online submission system

Fair and swift peer-review system

Efficient typesetting and proofreading procedure

Display of the result of downloads and visits, as well as the number of cited articles Maximum dissemination of your research work

Submit your manuscript at: http://papersubmission.scirp.org/

Or contact psych@scirp.org 